\title{
Double-Lumen Needle Follicular Flushing System versus Single-Lumen Aspiration Needle in IVF/ICSI Patients with Poor Ovarian Response: A Meta-Analysis
}

\author{
Weijie Xing*, Jianping Ou, Liuhong Cai, Xin Tao \\ Center for Reproductive Medicine, The Third Affiliated Hospital of Sun Yat-sen University, Guangzhou, China \\ Email: *838295988@qq.com
}

How to cite this paper: Xing, W.J., Ou, J.P., Cai, L.H. and Tao, X. (2017) Double-Lumen Needle Follicular Flushing System versus Single-Lumen Aspiration Needle in IVF/ICSI Patients with Poor Ovarian Response: A Meta-Analysis. Advances in Sexual Medicine, 7, 167-178.

https://doi.org/10.4236/asm.2017.74014

Received: September 11, 2017

Accepted: October 15, 2017

Published: October 18, 2017

Copyright $\odot 2017$ by authors and Scientific Research Publishing Inc. This work is licensed under the Creative Commons Attribution International License (CC BY 4.0).

http://creativecommons.org/licenses/by/4.0/

\section{Open Access}

\begin{abstract}
Background: The present study performed a meta-analysis to comprehensively analyze existing randomized controlled trials (RCT) involving the use of double-lumen needle in patients with poor ovarian response to explore whether double-lumen needle was good for specific patients. Methods: The PubMed, EMBASE, Cochrane Library databases and two randomized controlled trials registration centers were thoroughly searched until April 2017. The clinical outcomes of IVF/ICSI cycles were compared between two groups with double-lumen needle and single-lumen needle. Results: Four RCT studies were included in this present meta-analysis. The oocytes yield was similar in two groups ( $\mathrm{OR} 0.88,95 \% \mathrm{CI} 0.66-1.16 ; \mathrm{I}^{2}=4 \%$ ). The procedure time with double-lumen needle was significantly longer than that with single-lumen needle (IV $=1.98,95 \% \mathrm{CI} 0.95-3.00 ; \mathrm{I}^{2}=86 \%$ ). The fertilization rate with double-lumen needle was lower than that with single-lumen needle (OR 0.66 , 95\%CI $\left.0.44-0.97 ; \mathrm{I}^{2}=0 \%\right)$. There was no significant difference of live birth rate in two groups (OR $0.76,95 \% \mathrm{CI} 0.32-1.76 ; \mathrm{I}^{2}=41 \%$ ). Conclusion: Double-lumen needle could not benefit patients with a POR in terms of the number of oocytes retrieved, oocyte recovery rate, normal fertilization rate, clinical pregnancy rate, and live birth rate, compared with single-lumen needle.
\end{abstract}

\section{Keywords}

Double-Lumen Needle, Single-Lumen Needle, Poor Ovarian Response

\section{Background}

In the past 20 years, ultrasound-guided transvaginal oocyte retrieval (TVOR) has 
become the standard method of collecting oocytes in assisted reproductive technology (ART). This method is safer and more effective than the earlier laparoscopically assisted oocyte retrieval [1] [2]. The conventional application of TVOR involves a single-lumen oocyte retrieval needle. However, this could lead to oocyte retention within the follicles, especially in patients with poor ovarian response (POR) who have a lower number of follicles, which may result in the failure to collect oocytes. Therefore, a double-lumen oocyte retrieval needle was introduced to resolve this problem. When a double-lumen needle (DLN) is applied in oocyte retrieval, fluid is repeatedly injected into the follicle for flushing. Theoretically, follicular flushing should facilitate oocyte retrieval and should maximize the collection of oocytes [3] [4] [5] [6]. Nevertheless, with the development of oocyte retrieval technology, an increasing number of studies have found that skilled oocyte retrieval by direct aspiration can be used to obtain the vast majority of oocytes. Conversely, the use of a DLN for follicular flushing would prolong the operative time, affect the fertilization of oocytes, and confer no benefit compared with the use of a single-lumen needle (SLN) [6] [7]. A meta-analysis published by Roque et al. in 2012 concluded that in an unselected group of patients, there was no advantage in using a DLN in oocyte retrieval [8]. Another meta-analysis also reported that for patients with a normal ovarian function, the use of a DLN in oocyte retrieval did not improve the patients' ART outcomes [9].

Is there a need to perform follicular flushing using a DLN in oocyte retrieval for patients with a POR with fewer follicles? What is the developmental potential of oocytes obtained via follicular flushing? These questions await further investigation. The present study performed a meta-analysis to comprehensively analyze existing randomized controlled trials (RCTs) involving the use of DLNs in patients with a POR to explore whether DLNs are advantageous to specific patients.

\section{Method}

\section{Literature Search}

A comprehensive search was performed on the PubMed database, EMBASE database, Cochrane Library, and clinical trial registries (WHO International Clinical Trials Registry Platform and Clinical Trials.gov). The search was performed using the following subject headings or keywords: "double-lumen needle," "follicular flushing," "IVF," "ICSI," and "ART." The searching time span was 1916-2017, and the end date of the search was April 2017. The search strategy followed the Preferred Reporting Items for Systematic Reviews and MetaAnalyses statement.

\section{Outcome Measures}

The main outcome measures included the following: number of oocytes retrieved, operative time of oocyte retrieval, fertilization rate, clinical pregnancy rate, and live birth rate. 


\section{Study Selection}

The inclusion criteria for the studies were as follows: 1) English studies; 2) study type: RCTs; and 3) study population: patients with a POR receiving IVF/ICSI treatments. The exclusion criteria were as follows: 1) reviews, conference abstracts, or meeting speeches; 2) studies with duplicate data; and 3) all studies with incomplete data.

\section{Data Extraction and Quality Assessment}

Two evaluators screened the studies independently based on the inclusion and exclusion criteria, which involved the following three steps: 1) Removal of duplicate studies using the End Note software; 2) Exclusion of studies that did not meet the inclusion criteria after reading the title and abstract of each study; 3 ) Screening of the entire study on the basis of the inclusion and exclusion criteria. Discrepancies in opinion were resolved by discussion or consulting with relevant experts.

The two evaluators extracted the study information independently. The extracted information included the following: first author, year of publication, study duration, experimental design, intervention measures, control measures, sample size, and outcome measures. They also crosschecked the information extracted from the other party, and discrepancies were resolved by joint discussion or by consulting with relevant experts.

The two evaluators evaluated the studies according to the Cochrane Handbook for Systematic Reviews of Interventions [10]. Six related domains were assessed in each included trial: 1) random sequence generation; 2) allocation concealment; 3) blinding of participants and personnel; 4) incomplete outcome data; 5) selective reporting; 6) other bias. Each item was judged as a rating of "low risk", "unclear risk" and "high risk" of bias.

\section{Statistical Analysis}

The meta-analysis was performed using the Review Manager (RevMan) 5.3 software. $\mathrm{I}^{2}>50 \%$ indicates a high heterogeneity, and a random effects model was used for the analysis. $\mathrm{I}^{2} \leq 50 \%$ indicates a low to moderate heterogeneity, and a fixed effects model was used for the analysis. $P<0.05$ indicates that the difference was statistically significant.

Sensitivity analyses were performed to explore possible explanations for heterogeneity and to examine the influence of various exclusion criteria on the overall risk estimate. We investigated the influence of a single study on the overall risk estimate by omitting one study each time. Publication bias was assessed by funnel plots [11].

\section{Results}

\section{Literature Search}

A total of 41 articles were extracted. After screening by titles and abstracts, we got 20 articles. 16 articles were excluded by assessment for eligibility (Not in 
POR: 10; Not a RCT: 2). Finally, 4 RCT studies were included in this present meta-analysis. The process for literature search was shown in Figure 1.

\section{Study Characteristics}

Characteristics of all studies from 2009-2017 included in the meta-analysis were summarized in Table 1 . The fertility method was intracytoplasmic sperm
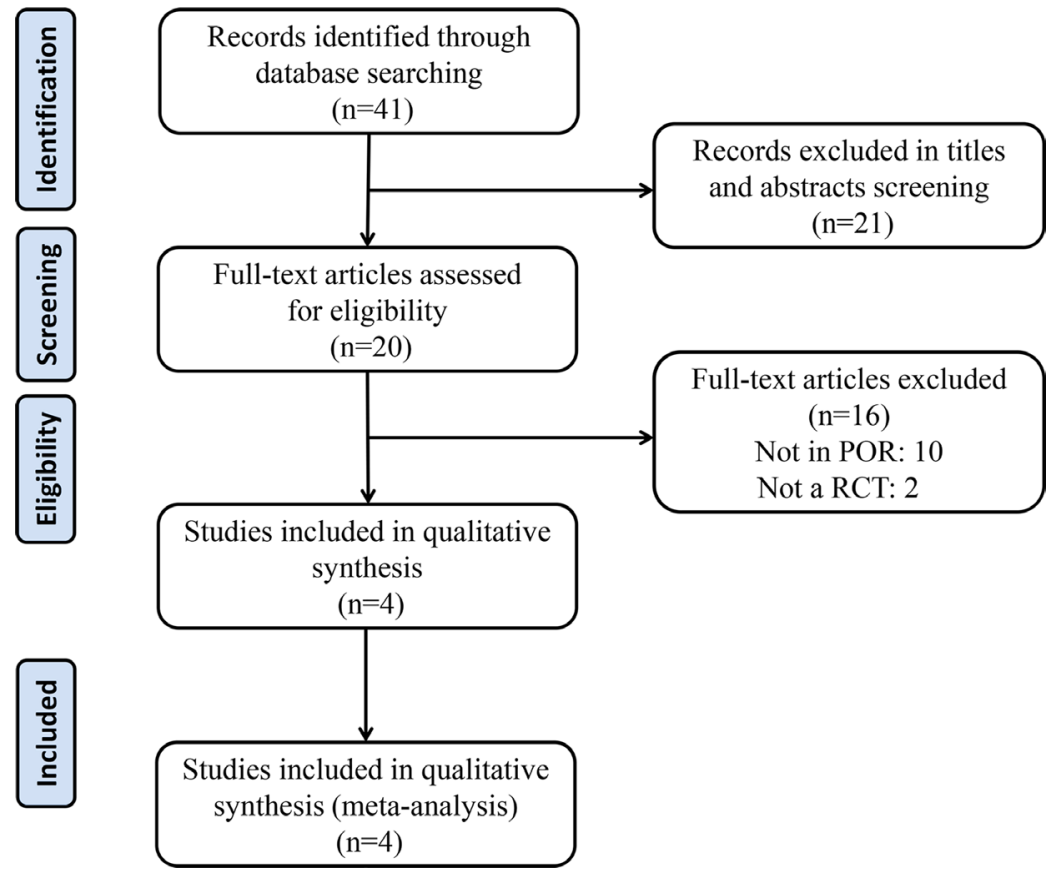

Full-text articles excluded $(n=16)$

Not in POR: 10

Not a RCT: 2

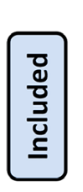

Studies included in qualitative $(\mathrm{n}=4)$

Figure 1. The process for literature search.

Table 1. Characteristics of all studies included in the systematic review.

\begin{tabular}{|c|c|c|c|c|c|c|c|}
\hline Study ID & Journal & Location & Duration of trial & Samples size & Interventions & Outcomes & conclusion \\
\hline $\begin{array}{l}\text { Haydardedeoglu } \\
2017\end{array}$ & BJOC & Turkey & 2015.01-2015.06 & $\begin{array}{l}\text { SLN-Group:40 } \\
\text { DLN-Group:40 }\end{array}$ & $\begin{array}{l}\text { SLN-Group: } 17-\text { G needle with single } \\
\text { lumen (Cook) } \\
\text { DLN-Group: } 17-\text { G needle with double } \\
\text { lumen (Cook); follicular flushing with } 10 \\
\text { mL culture medium for three times }\end{array}$ & $\begin{array}{l}\text { No. of oocytes } \\
\text { retrieved/No. of } \\
\text { follicles aspirated, No. } \\
\text { of MII oocyte, } \\
\text { procedure time, FR, } \\
\text { IR, PR, LBR }\end{array}$ & $\begin{array}{l}\text { DLN is time consuming } \\
\text { and has similar results } \\
\text { comparing to SLN. }\end{array}$ \\
\hline Horn 2017 & $\begin{array}{l}\text { Hum } \\
\text { Reprod }\end{array}$ & Germany & $2015.02-2016.03$ & $\begin{array}{l}\text { SLN-Group:39 } \\
\text { DLN-Group:39 }\end{array}$ & $\begin{array}{l}\text { SLN-Group: } 17-G \text { needle with single } \\
\text { lumen (Gynetics }{ }^{\circledR} \text { ) } \\
\text { DLN-Group: } 17-\text { G Steiner-Tan Needle }{ }^{\circledR} \\
\text { system with follicular flushing for three } \\
\text { times }\end{array}$ & $\begin{array}{l}\text { No. of oocytes } \\
\text { retrieved/No. of } \\
\text { follicles aspirated, } \\
\text { procedure time, } \\
\text { OPR/LBR, }\end{array}$ & $\begin{array}{l}\text { The use of DLN is } \\
\text { unlikely to benefit the } \\
\text { prognosis of patients } \\
\text { with poor ovarian } \\
\text { response. }\end{array}$ \\
\hline Levens 2009 & $\begin{array}{l}\text { Fertil } \\
\text { Steril }\end{array}$ & USA & 2007.04-2007.10 & $\begin{array}{l}\text { SLN-Group:15 } \\
\text { DLN-Group:15 }\end{array}$ & $\begin{array}{l}\text { SLN-Group: } 16-G \text { Echotip }{ }^{\circledR} \text { ovum } \\
\text { aspiration needle with single lumen } \\
\text { (Cook, K-J-ANC-16R-35) } \\
\text { DLN-Group:16-G double lumen needles } \\
\text { (Cook, K-OPSD-1635-B-S); follicular } \\
\text { flushing with } 2 \mathrm{~mL} \text { sterile PBS once }\end{array}$ & $\begin{array}{l}\text { No. of oocytes } \\
\text { retrieved/No. of } \\
\text { follicles aspirated, No. } \\
\text { of MII oocyte, } \\
\text { procedure time, FR, } \\
\text { OPR }\end{array}$ & $\begin{array}{l}\text { The results did not } \\
\text { demonstrate improved } \\
\text { oocyte recovery with } \\
\text { DLN even among those } \\
\text { most likely to benefit } \\
\text { from its application. }\end{array}$ \\
\hline Mok-Lin 2013 & $\begin{array}{l}\text { Hum } \\
\text { Reprod }\end{array}$ & USA & 12 -month period & $\begin{array}{l}\text { SLN-Group:25 } \\
\text { DLN-Group:25 }\end{array}$ & $\begin{array}{l}\text { SLN-Group: } 16-G \text { Echotip }{ }^{\circledR} \text { ovum } \\
\text { aspiration needle with single lumen } \\
\text { (Cook) } \\
\text { DLN-Group: 16-G Echotip }{ }^{\circledR} \text { ovum } \\
\text { aspiration needle with double lumen } \\
\text { (Cook); follicular flushing with } 5 \mathrm{~mL} \\
\text { culture medium for four times }\end{array}$ & $\begin{array}{l}\text { No. of oocytes } \\
\text { retrieved/No. of } \\
\text { follicles aspirated, No. } \\
\text { of MII oocyte, } \\
\text { procedure time, FR, } \\
\text { IR, PR, LBR }\end{array}$ & $\begin{array}{l}\text { DLN in the poorest } \\
\text { responders does not } \\
\text { increase the number of } \\
\text { oocytes retrieved and } \\
\text { may result in lower } \\
\text { implantation and clinical } \\
\text { pregnancy rates. }\end{array}$ \\
\hline
\end{tabular}


injection (ICSI) in two studies and IVF/ICSI combination in two studies [5] [12]. There were 2 studies with embryo transferred on day 3 [12] [13], 2 studies without mentioned it. The main outcomes were oocytes yield (No. of oocytes retrieved/No. of follicles aspirated), procedure time. The fertilization rate (FR) were compared in 3 studies [5] [12] [13]. The live birth rate (LBR) were compared in 3 studies [12] [13] [14].

Systematic risk assessment of methodological bias of included RCTs revealed two RCTs [5] [14] did not clearly describe "blinding of participants and personnel (performance bias)" and two RCTs [13] [14] did not clearly describe "blinding of outcome assessment (detection bias), therefore, we rated them at unclear risk of bias. We rated two studies [5] [14] at unclear risk in attribution bias, because we were unable to determine the integrity of data. We assessed one study [13] at an unclear risk of other bias, for an insufficient information about basic characters (Figure 2 and Figure 3).

\section{Meta-analysis}

\section{Oocytes yield}

All of the four RCT studies reported the data about oocytes yield (No. of oocytes retrieved/No. of follicles aspirated) [5] [12] [13] [14]. As shown in Figure 4 , the oocytes yield was similar in two groups (OR $0.88,95 \%$ CI $0.66-1.16 ; \mathrm{I}^{2}=$ $4 \%)$.

\section{Procedure time}

All of the four RCT studies reported the data about procedure time [5] [12] [13] [14]. From Figure 5, we found that the procedure time with double-lumen needle was significantly longer than that with single-lumen needle (IV $=1.98$, 95\%CI 0.95 - 3.00; $\mathrm{I}^{2}=86 \%$ ).

\section{Fertilization rate}

Only three RCT studies reported the data of fertilization rate [5] [12] [13]. In all of the three RCT studies, the fertilization rates with double-lumen needle were all lower than those with single-lumen needle. After meta-analysis, we found that the fertilization rate with double-lumen needle were all lower than that with single-lumen needle (OR 0.66, 95\%CI 0.44 - 0.97; $\mathrm{I}^{2}=0 \%$; Figure 6).

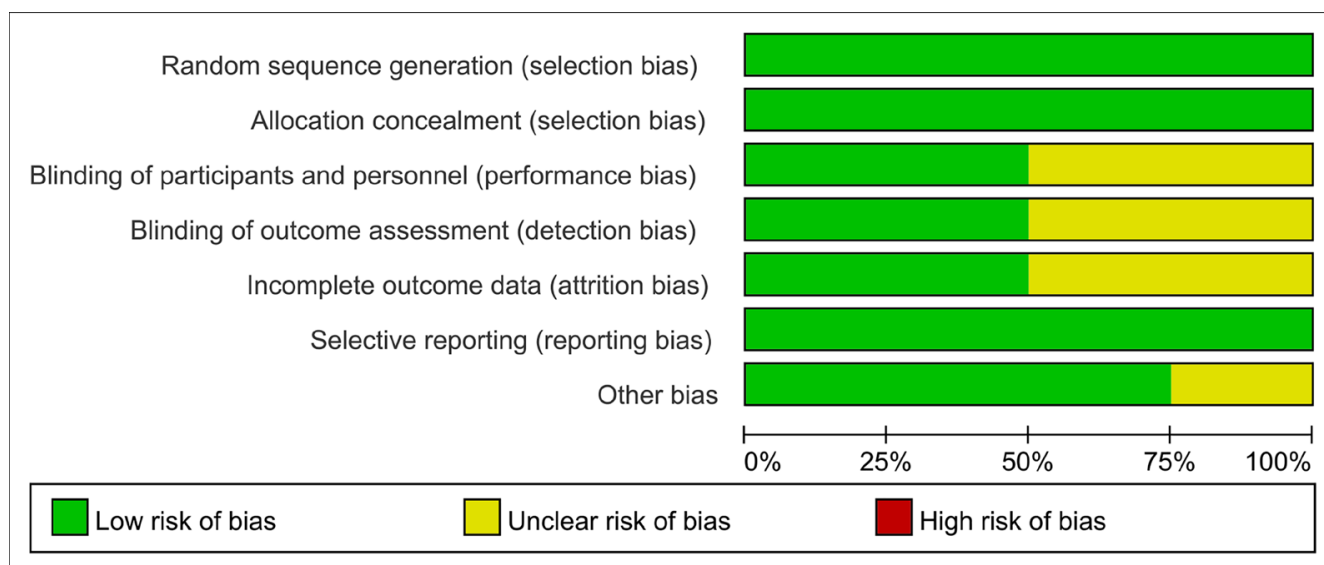

Figure 2. Risk of bias graph. 


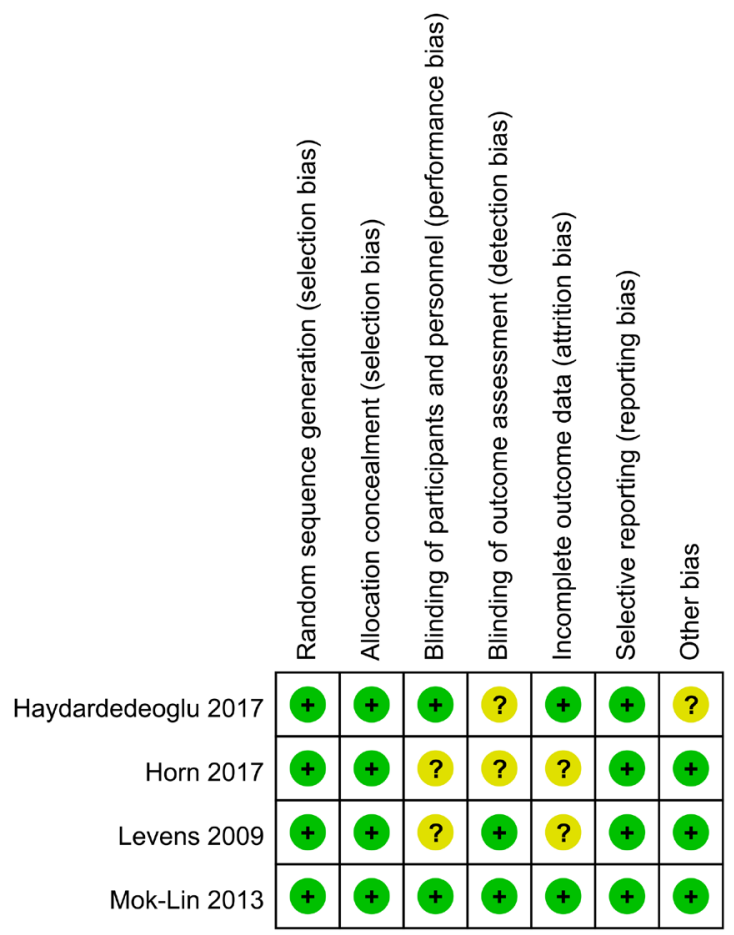

Figure 3. Risk of bias summary. "+" means "low risk of bias"; "?” means "unclear risk of bias".

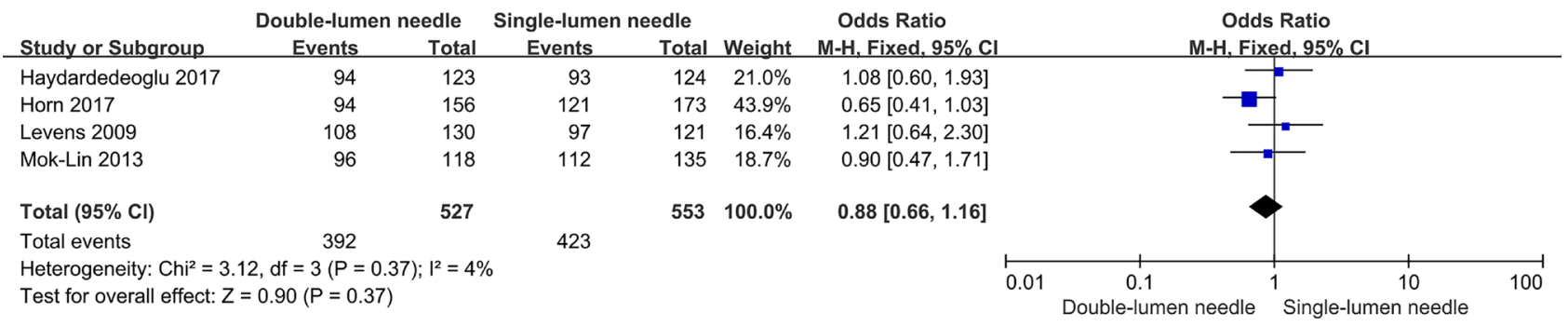

Figure 4. Forest plot of oocytes yield with DLNs versus SLNs.

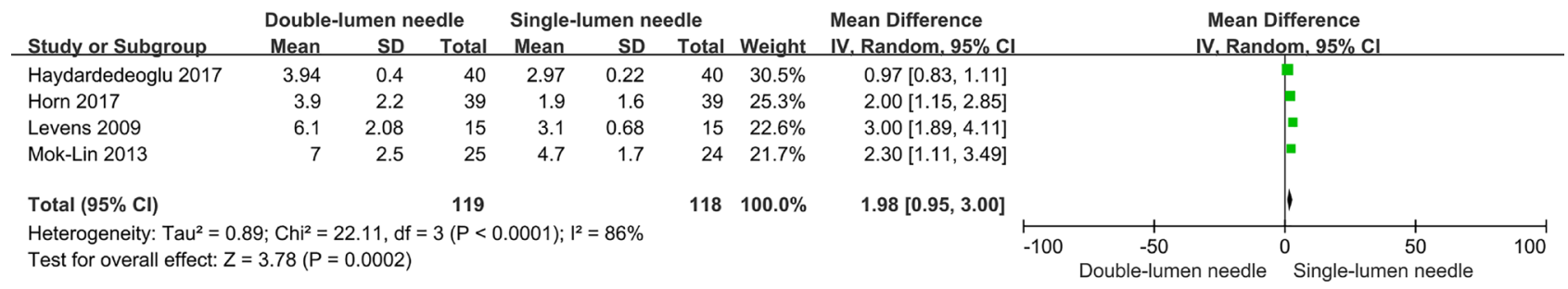

Figure 5. Forest plot of procedure time with DLNs versus SLNs.

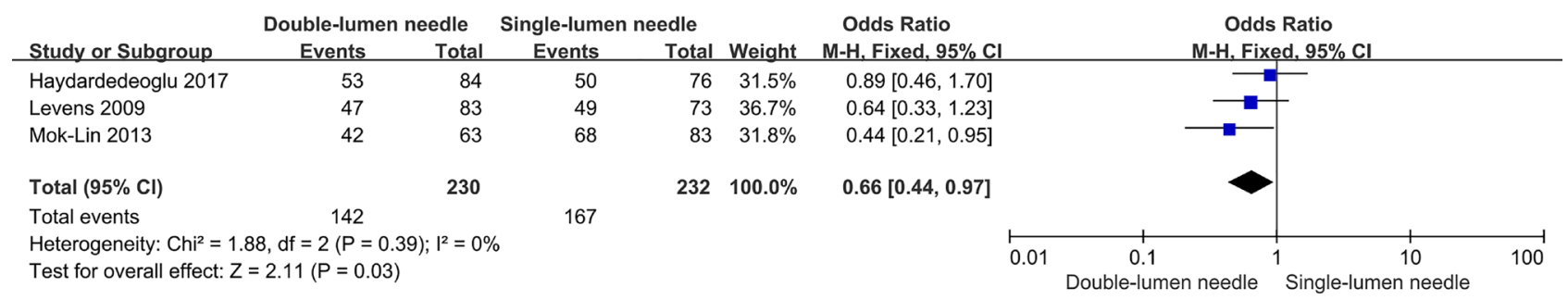

Figure 6. Forest plot of fertilization rate with DLNs versus SLNs. 


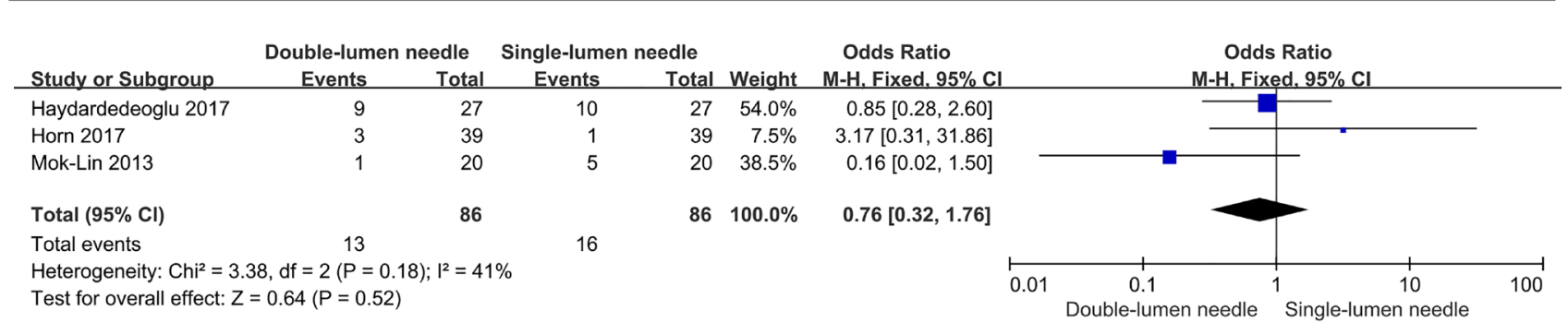

Figure 7. Forest plot of live birth rate with DLNs versus SLNs.

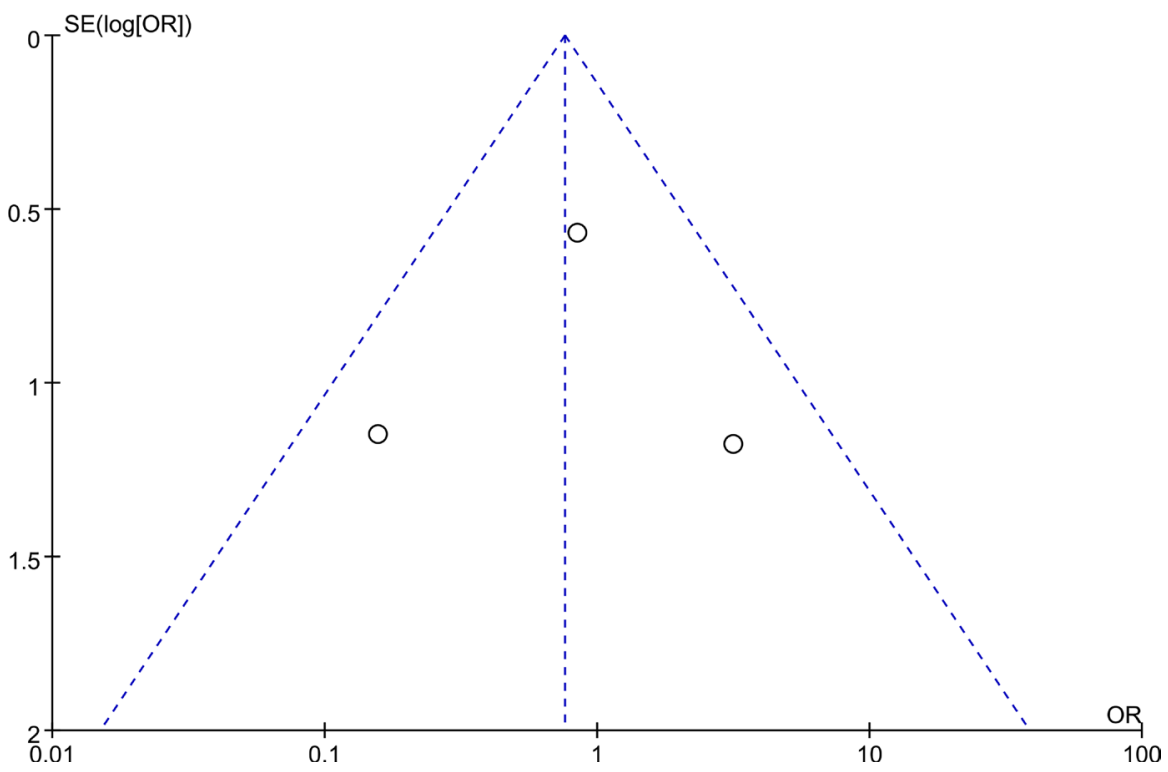

Figure 8. Funnel plot illustrating publication bias. All studies lie inside the 95\% CIs, with an even distribution around the vertical, indicating no obvious publication bias.

\section{Live birth rate}

Three out of four RCT studies reported the live birth rate (LBR) [12] [13] [14]. The LBR were lower in two studies with double-lumen needle than that with singlelumen needle [12] [13]. Only one study showed that the LBR was higher with double-lumen needle [14]. After meta-analysis, there were no significant difference of LBR in two groups (OR 0.76, 95\%CI $0.32-1.76$; $\mathrm{I}^{2}=41 \%$; Figure 7).

\section{Sensitivity Analysis and Publication Bias}

We investigated the influence of a single study on the overall risk estimate by omitting one study each time. In the sensitivity analysis, the exclusion of any one study did not materially alter the overall $\mathrm{P}$ value or heterogeneity $\left(\mathrm{I}^{2}\right)$.

Figure 8 shows a funnel plot of the studies included in this meta-analysis. All studies lie inside the $95 \%$ CIs, with an even distribution around the vertical, indicating no obvious publication bias.

\section{Discussion}

The aim of in vitro fertilization-embryo transfer (IVF-ET) is to collect highquality oocytes at the minimum cost, thus obtaining high-quality embryos to improve clinical pregnancy rates. The majority of fertility centers in China and 
abroad have stopped using follicular flushing in patients with a normal ovarian response. However, follicular flushing is still applied to patients with a POR with the aim of increasing the number of oocytes retrieved. The reduced ovarian function in patients with a POR often leads to the increased dosage of gonadotropins (Gn), long duration of $\mathrm{Gn}$ stimulation, and fewer mature follicles. In ART, difficulty in oocyte retrieval is the most common problem that occurs during oocyte collection in these patients. The number of oocytes retrieved can directly affect their pregnancy outcomes, and surgeons often hope that mature ova can be obtained by repeated follicular flushing.

The results of the meta-analysis performed in this study indicate that compared with patients with a POR who used SLNs for oocyte retrieval, patients who used DLNs did not show a higher total number of oocytes retrieved or a significant increase in oocyte retrieval rates, but had a significantly longer operative time. A longer operative time would surely increase the patients' suffering and perioperative risk [9].

Furthermore, further analyses on the quality and developmental potential of the oocytes indicated that oocytes retrieved using DLNs showed significantly reduced normal fertilization rates and clinical pregnancy rates compared with those retrieved using SLNs; the live birth rate showed different extents of reduction. The possible reasons for these observations include the following:

1) Influence of the number of flushing. The follicular flushing fluid comes into direct contact with the ovum. Moreover, the ovum is extremely sensitive to the temperature, $\mathrm{pH}$, osmotic pressure, and other factors in the external environment, whereby minor variations could lead to irreversible changes. Therefore, attention should be paid to the temperature of the flushing fluid and the sterile condition of the operation during follicular flushing. Repeated follicular flushing will not only increase the duration of transvaginal puncture, but also prolong the duration of oocyte immersion and exposure to the flushing fluid and increase the rate of oocyte morphological abnormalities. Studies have shown that approximately $40 \%$ of oocytes can be retrieved via direct aspiration; $82 \%$ can be retrieved after two times of follicular flushing; $97 \%$ can be retrieved after three times of follicular flushing; the oocyte retrieval rate decreases after more than three times of flushing [15] [16]. Neyens et al. [17] showed that the cumulusoocyte complex could be observed in approximately $90 \%$ of oocytes obtained by follicular flushing of up to two times, which did not affect the fertilization rate, pregnancy rate, and embryo quality. However, excessive follicular flushing significantly increased the rate of oocyte morphological abnormalities. Hussein compared the oocyte viability, cleavage rate after fertilization, and other outcome measures between oocytes recovered under transvaginal ultrasoundguided follicular puncture aspiration and in subsequent flushing after follicular aspiration. They found that the recovery rate and quality of oocytes retrieved under transvaginal ultrasound-guided follicular puncture and up to $4 \mathrm{~mL}$ of flushes were suitable for IVF. There was no need for repeated follicular flushing.

During the process of follicular growth, the follicular cavity will continue to 
expand; the follicular fluid will continue to increase, while the oocyte and granulosa cells will be pushed to one side to form the cumulus. Under natural conditions, the pre-ovulatory oocyte will eventually detach itself from the follicular wall and be suspended in the follicular cavity. We can infer from this that less mature oocytes will be more tightly bound to the follicular wall. Therefore, in theoretical terms, flushing via DLNs can be used to obtain a proportion of immature oocytes that are bound more tightly, thus increasing the oocyte retrieval rate. However, this would reduce the ratio of MII oocytes and lower the normal fertilization rate [16].

2) Flushing pressure: It is generally believed that the higher negative pressure during the oocyte retrieval process will damage the oocyte quality [16] [18].

3) Excessive flushing will also increase the probability of infection. This not only causes harm to the patients, but may also lead to pathogen contamination of the oocytes and embryos, thus causing the patients to have no viable embryos.

4) Patients with a POR themselves have fewer oocyte and embryo qualities; hence, follicular flushing is unable to change the essence of their poor-quality oocytes. Conversely, it may lead to the retrieval of oocytes with lower levels of maturity, thus leading to poorer IVF laboratory outcomes.

5) Lastly, follicular flushing would also aspirate more granulosa cells, leading to the excessive depletion of granulosa cells in the follicle. This could affect the functioning of the corpus luteum, thus influencing the pregnancy rates.

The Revman 5.3 software was used in this study to analyze the operative time, which found that the studies had a high heterogeneity $\left(\mathrm{I}^{2}=86 \%\right)$. Further analysis of the included studies revealed that the type and model of the DLNs, number of flushes, and volume of flushing fluid used in each study were different. These factors may influence the operative time, thus causing the studies to have a slightly high level of heterogeneity. The meta-analysis of the other outcome measures, such as the number of oocytes retrieved, oocyte recovery rate, fertilization rate, clinical pregnancy rate, and live birth rate, did not indicate the presence of significant heterogeneity. The sensitivity analysis was performed by removing each study individually, and there was no significant decrease in heterogeneity when any study was removed.

This study has the following limitations: 1) The time span of the studies included was relatively long (from 2009 to 2017). There have been rapid developments in ART during this period, with major advancements in controlled ovarian hyper-stimulation protocols, embryo culture techniques, embryo cryopreservation techniques, transfer techniques, and other areas. Therefore, the results of the studies will be biased. 2) The included studies originated from different countries and fertility centers. 3) The sample sizes of the included RCTs were relatively small. 4) Lastly, the literature search may have been incomplete.

\section{Conclusion}

In summary, a meta-analysis was performed in this study to examine whether 
DLNs could not benefit patients with a POR in terms of the number of oocytes retrieved, oocyte recovery rate, normal fertilization rate, clinical pregnancy rate, and live birth rate, compared with SLNs.

\section{Competing Interests}

The authors declare that they have no competing interests.

\section{References}

[1] Lenz, S., Lauritsen, J.G. and Kjellow, M. (1981) Collection of Human Oocytes for In Vitro Fertilisation by Ultrasonically Guided Follicular Puncture. Lancet, 1, 1163-1164. https://doi.org/10.1016/S0140-6736(81)92335-7

[2] Ludwig, A.K., Glawatz, M., Griesinger, G., Diedrich, K. and Ludwig, M. (2006) Perioperative and Post-Operative Complications of Transvaginal Ultrasound-Guided Oocyte Retrieval: Prospective Study of $>1000$ Oocyte Retrievals. Human Reproduction, 21, 3235-3240. https://doi.org/10.1093/humrep/del278

[3] el Hussein, E., Balen, A.H. and Tan, S.L. (1992) A Prospective Study Comparing the Outcome of Oocytes Retrieved in the Aspirate with Those Retrieved in the Flush during Transvaginal Ultrasound Directed Oocyte Recovery for In-Vitro Fertilization. British Journal of Obstetrics and Gynaecology, 99, 841-844. https://doi.org/10.1111/j.1471-0528.1992.tb14417.x

[4] Hill, M.J. and Levens, E.D. (2010) Is There a Benefit in Follicular Flushing in Assisted Reproductive Technology? Current Opinion in Obstetrics and Gynecology, 22, 208-212. https://doi.org/10.1097/GCO.0b013e3283373bfe

[5] Levens, E.D., Whitcomb, B.W., Payson, M.D. and Larsen, F.W. (2009) Ovarian Follicular Flushing among Low-Responding Patients Undergoing Assisted Reproductive Technology. Fertility and Sterility, 91, 1381-1384. https://doi.org/10.1016/j.fertnstert.2008.04.034

[6] Wongtra-Ngan, S., Vutyavanich, T. and Brown, J. (2010) Follicular Flushing during Oocyte Retrieval in Assisted Reproductive Techniques. The Cochrane Database of Systematic Reviews, CD004634.

[7] Tan, S.L., Waterstone, J., Wren, M. and Parsons, J. (1992) A Prospective Randomized Study Comparing Aspiration Only with Aspiration and Flushing for Transvaginal Ultrasound-Directed Oocyte Recovery. Fertility and Sterility, 58, 356-360. https://doi.org/10.1016/S0015-0282(16)55230-3

[8] Roque, M., Sampaio, M. and Geber, S. (2012) Follicular Flushing during Oocyte Retrieval: A Systematic Review and Meta-Analysis. Journal of Assisted Reproduction and Genetics, 29, 1249-1254. https://doi.org/10.1007/s10815-012-9869-9

[9] Levy, G., Hill, M.J., Ramirez, C.I., Correa, L., Ryan, M.E., DeCherney, A.H., et al. (2012) The Use of Follicle Flushing during Oocyte Retrieval in Assisted Reproductive Technologies: A Systematic Review and Meta-Analysis. Human Reproduction, 27, 2373-2379. https://doi.org/10.1093/humrep/des174

[10] Zhang, B., Cui, L., Tang, R., Ding, L., Yan, L. and Chen, Z.J. (2017) Reduced Ectopic Pregnancy Rate on Day 5 Embryo Transfer Compared with Day 3: A Meta-Analysis. PLoS One, 12, e0169837. https://doi.org/10.1371/journal.pone.0169837

[11] Egger, M., Davey, S.G., Schneider, M. and Minder, C. (1997) Bias in Meta-Analysis Detected by a Simple, Graphical Test. BMJ, 315, 629-634.

https://doi.org/10.1136/bmj.315.7109.629 
[12] Mok-Lin, E., Brauer, A.A., Schattman, G., Zaninovic, N., Rosenwaks, Z. and Spandorfer, S. (2013) Follicular Flushing and in Vitro Fertilization Outcomes in the Poorest Responders: A Randomized Controlled Trial. Human Reproduction, 28, 2990-2995. https://doi.org/10.1093/humrep/det350

[13] Haydardedeoglu, B., Gjemalaj, F., Aytac, P.C. and Kilicdag, E.B. (2017) Direct Aspiration versus Follicular Flushing in Poor Responders Undergoing Intracytoplasmic Sperm Injection: A Randomised Controlled Trial. BJOG, 124, 1190-1196. https://doi.org/10.1111/1471-0528.14629

[14] Von Horn, K., Depenbusch, M., Schultze-Mosgau, A. and Griesinger, G. (2017) Randomized, Open Trial Comparing a Modified Double-Lumen Needle Follicular Flushing System with a Single-Lumen Aspiration Needle in IVF Patients with Poor Ovarian Response. Human Reproduction, 32, 832-835.

https://doi.org/10.1093/humrep/dex019

[15] Bagtharia, S. and Haloob, A.R. (2005) Is There a Benefit from Routine Follicular Flushing for Oocyte Retrieval? Journal of Obstetrics and Gynaecology, 25, 374-376. https://doi.org/10.1080/01443610500118970

[16] Kara, M., Aydin, T. and Turktekin, N. (2012) Is Follicular Flushing Really Effective? A Clinical Study. Archives of Gynecology and Obstetrics, 286, 1061-1064. https://doi.org/10.1007/s00404-012-2424-1

[17] Neyens, S., De Neubourg, D., Peeraer, K., De Jaegher, N., Spiessens, C., Debrock, S., et al. (2016) Is There a Correlation between the Number of Follicular Flushings, Oocyte/Embryo Quality and Pregnancy Rate in Assisted Reproductive Technology Cycles? Results from a Prospective Study. Gynecologic and Obstetric Investigation, 81, 34-40. https://doi.org/10.1159/000434750

[18] Mehri, S., Levi Setti, P.E., Greco, K., Sakkas, D., Martinez, G. and Patrizio, P. (2014) Correlation between Follicular Diameters and Flushing versus No Flushing on Oocyte Maturity, Fertilization Rate and Embryo Quality. Journal of Assisted Reproduction and Genetics, 31, 73-77. https://doi.org/10.1007/s10815-013-0124-9 


\section{Abbreviation Note List}

TVOR ultrasound-guided trans-vaginal oocyte retrieval

ART assisted reproductive technology

POR poor ovarian response

DLN double-lumen needle

SLN single-lumen needle

RCT randomized controlled trials

IVF-ET In vitro fertilization-embryo transfer

ICSI intracytoplasmic sperm injection

FR fertilization rate

IR Implantation rate

PR Pregnancy rate

OPR Ongoing pregnancy rate

LBR live birth rate

Gn gonadotropins 